Essay Review

\title{
Les systèmes hospitaliers contemporains, entre histoire sociale des techniques et business history
}

Pierre-Yves Donzé

Keywords: hospital history; social history of techniques; business history; health economy; hospital system

L'histoire des hôpitaux est un champ historiographique ancien, qui s'est structuré au cours des années 1950 et 1960 dans le contexte du redéploiement architectural, organisationnel et technique des hôpitaux. La modernisation hospitalière de l'après-guerre a sans doute joué un rôle essentiel dans la naissance de cet intérêt pour l'histoire et le patrimoine hospitaliers, qui s'est cristallisé dans la fondation d'associations telles que le Centro Italiano di Storia Ospedaliera (1956), la Société française d'histoire des hôpitaux (1958) ou encore la Deutsche Gesellschaft für Krankenhausgeschichte (1964). Généralement dirigées par des administrateurs d'hôpitaux et des médecins intéressés à l'histoire de leur branche, ces sociétés ont stimulé la recherche sur l'histoire des hôpitaux, notamment grâce à des revues périodiques, comme le Bulletin de la Société française d'histoire des hôpitaux (1959) ou Historia Hospitalium. Mitteilungen der Deutschen Gesellschaft für Krankenhausgeschichte (1966). C'est dans ce contexte qu'ont été réalisées de nombreuses monographies d'établissements hospitaliers et que sont nées les premières synthèses en histoire des hôpitaux ${ }^{1}$. De manière générale, ces travaux appréhendent le développement hospitalier sur le long terme (de l'Ancien Régime, voire du Moyen Age, au XX $\mathrm{XX}^{\mathrm{e}}$ siècle) et l'expliquent comme une conséquence directe, quasi mécanique, et inéluctable, de l'essor des connaissances médicales, de la complexification des techniques mises en œuvre, des nouvelles découvertes scientifiques, bref de la marche vers une médecine triomphante débouchant sur des hôpitaux plus grands et mieux équipés.

1 Voir par exemple Imbert 1982.

Dr Pierre-Yves Donzé, Institut d'histoire, Université de Neuchâtel, Espace Louis-Agassiz 1, CH-2001 Neuchâtel (pierre-yves.donze@unine.ch). 


\section{L'histoire des hôpitaux sous l'emprise de la médicalisation}

Une telle perspective s'avère toutefois insuffisante et peu convaincante. La médecine ne s'est en effet pas constituée et ne se développe pas de manière linéaire et constante. Elle n'entretient pas non plus de liens de cause à effet avec les établissements hospitaliers dans lesquels elle se déploie. Depuis les années 1970, les historiens se sont attachés à faire intervenir le jeu des acteurs et montrer que la construction de la médecine hospitalière ne répond pas uniquement à des impératifs scientifiques, intrinsèques au savoir médical, mais aussi à des questions d'ordre politique, social et économique. Les fonctions de l'hôpital dans la cité sont en effet multiples et dépassent le seul cadre des découvertes médicales. Pour l'essentiel, cette nouvelle histoire de la médecine s'est structurée autour de la «médicalisation», un concept fondé par Michel Foucault, lorsqu'il aborde la question de la réorganisation des hôpitaux à la fin du XVIII ${ }^{\mathrm{e}}$ siècle. Pensée comme le recentrage de ces établissements sur la prise en charge de malades et l'intervention croissante de médecins, la médicalisation est alors considérée comme une conséquence de «l'introduction de mécanismes disciplinaires dans l'espace désordonné de l'hôpital»². Cette approche a très largement marqué, voire inspiré, les historiens, notamment anglo-saxons ${ }^{3}$, qui à leur tour ont marqué les recherches en histoire sociale de la médecine, à l'image de celles menées par Othmar Keel dans ses travaux sur la transformation des hôpitaux en des centres de pratique médicale et d'enseignement clinique. Ce dernier fait de la médicalisation hospitalière une étape déterminante de l'affirmation du paradigme anatomo-clinique ${ }^{4}$ et la définit dans ce contexte comme «la transformation [...] qui se produit au $18^{\mathrm{e}}$ siècle dans différents pays d'Europe, de certaines institutions d'assistance ou de régulation sociale en institutions thérapeutiques et médico-scientifiques» ${ }^{5}$. En Allemagne, l'influence de Foucault est aussi patente et les historiens font de la médicalisation une base essentielle de la disciplinarisation sociale (Soziale Disziplinierung) au cours du XIX ${ }^{\mathrm{e}}$ siècle. Le renforcement de la police sanitaire, l'essor des assurances-maladie et le développement des hôpitaux sont généralement interprétés comme autant d'éléments destinés à assurer un contrôle sur des classes populaires en voie de prolétarisation ${ }^{6}$.

2 Foucault 1998, 517. Pour une vision plus large de l'apport de Foucault à l'histoire des hôpitaux, voir Foucault 1963.

3 A propos de l'influence de Foucault sur les historiens anglo-saxons, voir Jones/Porter 1994. Pour la France, un bon exemple de l'influence de Foucault sur les monographies hospitalières est donné par Delamare/Delamare-Riche 1990.

4 Keel 2001.

5 Keel 2001,29sq.

6 Labisch/Spree 1997; Loetz 1993. 
La notion de médicalisation s'est toutefois passablement diversifiée au cours des années 1980 et 1990, à l'exemple des travaux réalisés par les historiens français Jacques Léonard, Jean-Pierre Goubert et Olivier Faure ${ }^{7}$, pour lesquels la médicalisation n'est pas uniquement un processus imposé par les classes dirigeantes sur le peuple, mais résulte d'une véritable révolution culturelle, dans le sens qu'elle repose en grande partie sur l'intériorisation par la population de nouvelles pratiques de soins. La médicalisation apparaît aujourd'hui comme un concept polysémique dont l'utilisation pose un certain nombre de problèmes. Elle n'est en effet que très rarement définie de manière explicite dans les divers travaux et le terme est utilisé pour définir des réalités fort diverses, sur une échelle chronologique très variable. De manière générale, on peut dire que la médicalisation exprime une idée de processus, qui fait passer d'un état avant à un état après, ce dernier se distinguant par une situation nouvelle dans laquelle la médecine occupe une position renforcée. Plusieurs types de processus ont été qualifiés de médicalisation par les historiens. Il peut s'agir par exemple d'innovations techniques (anesthésie, antisepsie, bactériologie, génétique, etc.), de la professionnalisation de certaines fonctions soignantes (médecins, personnel infirmier, sagesfemmes, etc.), de réorganisations institutionnelles (comme la séparation des malades et des vieillards dans les hôpitaux) ou de changement d'attitude de la population (comme le recours aux soins hospitaliers), autant d'éléments qui ont transformé la pratique de la médecine et la consommation de soins. Ainsi, on peut parler de médicalisation aussi bien dans le cas de la place grandissante que prend le corps médical dans certains hôpitaux urbains du $\mathrm{XVI}^{\mathrm{e}}$ siècle, que dans l'apparition de soucis hygiénistes à la fin du $\mathrm{XVIII}^{\mathrm{e}}$ siècle ou dans la rationalisation de l'organisation hospitalière au cours de l'entre-deux-guerres 8 . Olivier Faure met bien en lumière le caractère déterministe qui peut découler de l'utilisation de ce terme: «Repérée dès le Moyen Age par une présence plus grande des médecins, la médicalisation se serait imperturbablement développée sans cesse avec la médecine clinique, la médecine pastorienne puis l'ouverture à tous d'un hôpital jusque-là réservé aux pauvres.» ${ }^{9}$ Ainsi que le souligne Faure, ce qui pose problème, c'est bien que la médicalisation soit porteuse d'une conception linéaire, déterministe, voire téléologique, du développement de la médecine au cours des

7 Léonard 1978; Goubert 1982; Faure 1982. Sur les diverses approches françaises de la médicalisation, voir Faure 1998.

8 Olivier Faure affirme que «sous la plume des historiens, le terme de médicalisation désigne des réalités multiples, renvoie à des origines différentes et suscite des interprétations opposées». Faure 1998, 53.

9 Faure 1995, 322. 
derniers siècles, chaque innovation - et les nouveaux usages sociaux qu'elle implique - étant perçue comme une étape du «moins médicalisé» vers le «plus médicalisé». Or, la médecine, pas plus que d'autres domaines de la science et de la technique, n'a connu une évolution linéaire et finaliste telle que peut parfois le faire imaginer l'usage du terme de médicalisation.

\section{L'histoire sociale des techniques appliquée à la médecine contemporaine}

Les recherches menées en histoire des techniques, et plus largement en histoire économique, permettent d'envisager la construction de la médecine hospitalière selon une autre approche. La notion de «système technique» apparaît en effet d'un usage beaucoup plus adapté ${ }^{10}$. Selon l'historien des techniques français Bertrand Gille, les systèmes techniques découlent d'un équilibre entre les diverses techniques en interaction à un moment donné ${ }^{11}$. Dans cette perspective, l'analyse historique met en évidence des phases d'équilibre et des phases de réadaptation du système lorsqu'une nouvelle technique apparaît ou évolue. De plus, le système technique n'est pas autonome, mais lié à d'autres systèmes (économique, politique, social, etc.), qui ont leur propre logique, et avec lesquels il est en interaction constante. Gille n'en accorde pas pour autant un rôle primordial aux acteurs: chez lui, le système technique résulte plus d'un processus cumulatif de l'ensemble des techniques mises en œuvre à un moment donné que d'un processus constructiviste découlant du jeu des acteurs ${ }^{12}$. Il est ainsi tout à fait imaginable et aisé d'appliquer à la médecine le modèle de Gille. Le «système technique médical» pourrait ainsi s'appréhender comme l'ensemble des techniques médicales existant à un moment donné et regroupant des machines et des objets (tables d'opérations, pinces, bistouris, stéthoscopes, etc.), des gestes regroupés en corps de doctrine (réunis dans des ouvrages et enseignés dans des universités), des produits (chloroforme, catgut, cocaïne, médicaments, etc.), etc. L'apparition d'une nouvelle technique, comme l'anesthésie au milieu du $\mathrm{XIX}^{\mathrm{e}}$ siècle, la radiologie dans les années 1890 ou les antibiotiques dans les années 1940, viendrait alors rompre l'équilibre du système technique médical, obligeant ce dernier à une réadaptation. Une telle lecture, même si elle

10 Le concept de «système technique» a donné lieu à une multitude de travaux théoriques et de monographies sur lesquels il n'y pas lieu de s'étendre exhaustivement dans le cadre de ce travail, qui n'est qu'un exemple de système technique appliqué à la médecine. Pour une première approche des systèmes techniques en histoire économique, voir les références citées dans la bibliographie de Caron 1997.

11 Voir Gille 1978.

12 Cohen/Pestre 1998, 735-738. 
prend en compte l'interaction avec d'autres systèmes (politique, économique, social, etc.), n'est toutefois pas suffisante. Le risque est grand en effet d'accorder une importance trop considérable à la technique médicale ellemême dans l'explication de la construction de la médecine hospitalière, le recours au concept de Gille ne faisant en fin de compte que renforcer l'interprétation évolutionniste.

La notion de système technique conceptualisée par Gille dans les années 1970 a grandement évolué, notamment grâce aux réflexions de sociologues constructivistes tels que Thomas Hughes. Ce dernier fait éclater les limites du système technique dans lequel il intègre des composantes sociales, économiques, politiques, organisationnelles et gestionnaires, si bien que «les systèmes qu'il étudie ressortissent donc à l'action humaine et non à une pure et simple comptabilité matérielle» ${ }^{13}$. Le système technique tel qu'il l'entend n'est pas l'unique cumul de machines, d'outils, de connaissances et de gestes techniques, mais l'interaction entre ces éléments et des modes de gestion, des modèles organisationnels, des politiques de financement, des intérêts industriels, etc. Dans son étude sur la constitution et le développement des systèmes électriques, Hughes met bien en évidence qu'ils ne sont pas les produits «naturels» d'une évolution de type darwinien, mais le résultat du jeu des divers «constructeurs de système» (ingénieurs, politiciens, financiers, etc.) et de l'influence d'un environnement externe (politique, économique, géographique, etc.). Les systèmes techniques ne sont pas des produits autonomes, mais sont construits et dirigés par des acteurs. Cette approche rompt radicalement avec une logique qui faisait de l'évolution technique le résultat d'une accumulation de connaissances et du génie de quelques «grands inventeurs». Bien au contraire, l'équilibre et la réadaptation des systèmes techniques résultent d'abord du jeu des acteurs ${ }^{14}$. Hughes utilise ainsi le concept de «système socio-technique», c'est-à-dire d'un système qui contient des éléments techniques proprement dits et des éléments organisationnels ${ }^{15}$. L'économique, le politique et le social ne se réduisent pas à l'environnement qui interagit avec le système technique, comme c'est le cas chez Gille, mais sont considérés en eux-mêmes comme des éléments du système sociotechnique. Dans une même perspective, François Caron met en avant le problème du passage d'un système socio-technique à un autre et montre que certains dysfonctionnements ou certaines innovations, de nature technique, politique, économique ou autre, accélèrent l'instabilité inhérente à tout système technique et la recherche d'un nouvel équilibre: les révolutions

13 Cohen/Pestre 1998, 735.

14 Hughes 1993.

15 Hughes 1998. 
industrielles sont ainsi vues comme des périodes de profonds bouleversements qui voient le passage d'un système technique à un autre ${ }^{16}$.

Cette manière d'appréhender l'histoire des techniques et des sciences a directement influencé l'histoire de la médecine. Plusieurs sociologues et historiens ont en effet intégré la médecine à leurs réflexions sur l'innovation et le changement technique. Dans la foulée des travaux de chercheurs comme Thomas Hughes, Wiebe Bijker et Trevor Pinch lancés dans les années 1980 ${ }^{17}$, certains auteurs se sont intéressés au développement technique de la médecine et «il s'est affirmé une 〈nouvelle école〉 qui traite de la technologie médicale comme d'un nouvel élément de l'histoire sociale, politique et économique de la médecine $[\ldots] \gg^{18}$. Stuart Blume prend ainsi l'exemple de l'imagerie médicale (rayons X, scanners, etc.) pour montrer que le développement de la technologie médicale répond à un processus constructiviste, comme c'est le cas dans les autres secteurs économiques ${ }^{19}$. En France, il faut souligner les travaux de Bruno Latour sur Pasteur et la construction de la science dans les laboratoires, qui ont influencé toute une génération d'historiens des sciences et de la technique ${ }^{20}$.

Inspiré par les travaux de Bijker et des sociologues de l'innovation, John Pickstone inclut la médecine au sein d'un ensemble plus large qu'il nomme Science, Technology and Medicine (STM), en référence à l'acronyme STS (Science, Technology and Society) utilisé en sociologie des techniques. Il montre ainsi que l'évolution de la médecine dépend de la culture environnante, d'une société qui se transforme et s'industrialise, de lieux de formation intellectuelle qui changent, etc. Le lien entre médecine et société permet une histoire globalisante du développement de la médecine ${ }^{21}$. Plusieurs auteurs proches de Pickstone et des divers Wellcome Institutes for the History of Medicine, en Grande-Bretagne, ont étudié des aspects particuliers du développement de la médecine comme science et comme technique, dans une approche constructiviste ${ }^{22}$. L'histoire sociale de la médecine s'est ainsi érigée en champ de recherche autonome, avec ses meneurs (John Pickstone, Roy Porter, etc.), ses maisons d'édition (Routledge, Macmillan, etc.), ses revues (Social History of Medicine, Bulletin of the History of Medicine, etc.) et ses lieux de recherche privilégiés (Wellcome Institute).

16 Caron 1997.

17 Bijker/Hughes/Pinch 1999 et Bijker 1995.

18 Stanton 1999.

19 Blume 1992.

20 Latour/Woolgar 1979 et Latour 2001. De nombreux auteurs cités ci-dessous se réfèrent en effet à ces travaux.

21 Pickstone 2000.

22 Cooter/Harrison/Sturdy 1999; Stanton 2002; Löwy 1993. 
En Suisse, l'histoire sociale des techniques médicales est encore peu développée et il faut souligner avec bonheur l'excellente étude de Thomas Schlich sur le développement de l'ostéosynthèse comme méthode privilégiée dans le traitement des fractures au cours de l'après-guerre. En mettant en avant la dimension sociale et économique de cette innovation thérapeutique, il montre que l'adoption par le monde médical des diverses découvertes techniques résulte avant tout de rapports de force sociaux. L'ostéosynthèse doit en l'occurrence son existence à un groupe de jeunes chirurgiens alémaniques socialement déclassés - ils ne sont pas engagés dans une carrière universitaire - et désireux de s'affirmer dans le milieu chirurgical, qui trouvent des alliances dans le monde industriel pour imposer leurs innovations ${ }^{23}$. Dans la même perspective, Monika Dommann a publié en 2003 une thèse sur le développement de la radiologie en Suisse alémanique durant la première partie du $\mathrm{XX}^{\mathrm{e}}$ siècle. Adoptant une démarche d'histoire sociale des techniques fortement inspirée par les travaux de Bruno Latour, elle s'est notamment intéressée à la manière dont la radiologie se constitue en champ autonome de la médecine, avec par exemple sa propre infrastructure technique et ses filières de recrutement d'un personnel «spécialisé» ${ }^{24}$.

\section{Les systèmes hospitaliers et la Business History}

L'histoire sociale des techniques appliquée en médecine n'a encore que très peu intégré la dimension économique dans ses réflexions. L'écrasante majorité des études faites dans ce champ de recherche n'aborde pas le cadre matériel dans lequel s'opèrent les innovations techniques de la médecine. L'application de méthodes d'analyse et de conceptions issues du champ de l'histoire économique est encore relativement rare. Les quelques historiens à avoir franchi le pas sont essentiellement américains et britanniques.

Il est vrai que la formidable croissance démographique et la très forte urbanisation que connaissent les Etats-Unis à la fin du XIX ${ }^{\mathrm{e}}$ siècle débouchent sur une profonde restructuration des lieux de soins, tels que les dispensaires et les hôpitaux, afin d'assurer la prise en charge d'une population en constante croissance. Le formidable changement d'échelle dont sont l'objet les établissements hospitaliers américains vers 1900 implique une réforme gestionnaire et organisationnelle qui n'a pas échappé aux histo-

23 Schlich 2002. Cette perspective est similaire à celle adoptée par Takahiro Ueyama pour expliquer l'émergence de l'électrothérapie comme spécialité autonome en Grande-Bretagne dans les années 1880-1920. Ueyama 1997.

24 Dommann 2003. 
riens $^{25}$. Il faut aussi souligner ici l'impact sur la recherche historique des travaux d'Alfred Chandler sur les grandes entreprises américaines ${ }^{26}$ auxquels font explicitement référence plusieurs historiens de la médecine (Thomas Goebel $^{27}$, Joel Howell, Barbara Bridgman Perkins ${ }^{28}$, Neil Larry Shumsky ${ }^{29}$, Paul Starr ${ }^{30}$, Steve Sturdy ${ }^{31}$, etc.). Steve Sturdy affirme ainsi par exemple que ce ne sont pas tant les découvertes médicales qui ont influencé le développement de la médecine à la fin du XIX ${ }^{\mathrm{e}}$ siècle mais «que les plus importants changements de la pratique médicale doivent être trouvés dans ce qu'il est convenu d'appeler l'administration médicale» ${ }^{32}$. Prenant l'exemple de la ville de Sheffield, il voit dans les développements de la médecine au $\mathrm{XX}^{\mathrm{e}}$ siècle un souci de rationaliser un travail qui se divise de plus en plus, sur le modèle industriel dans lequel baigne la cité. Une approche similaire est adoptée par d'autres historiens britanniques, comme Roger Cooter dans son étude sur l'organisation de la chirurgie orthopédique au début du XX $\mathrm{XX}^{\mathrm{e}}$ siècle ${ }^{33}$ ou John Pickstone lorsqu'il aborde le développement des hôpitaux de Manchester ${ }^{34}$. Quant à Neil Larry Shumsky, il avance que «le monde de la médecine contemporaine est fait de structure, de bureaucratie et d'organisation $»^{35}$ et explique le fonctionnement de la Clinique municipale de San Francisco en se fondant sur les travaux de Chandler, tandis que pour Barbara Bridgman Perkins, la restructuration des hôpitaux américains dans les années 1900-1930 se fonde sur des méthodes organisationnelles importées du secteur industriel (division du travail) qui permettent l'affirmation de spécialités médicales. Le travail le plus convaincant sur le rôle de l'administration et du management dans l'industrialisation de la médecine hospitalière reste néanmoins l'ouvrage classique et largement cité de Joel Howel1 ${ }^{36}$. En se basant sur les dossiers de patients de deux hôpitaux américains - le New York Hospital et le Pennsylvania Hospital de Philadelphie - dans les années 1900-1925, il montre comment la technologie médicale s'affirme au sein des établissements hospitaliers et déclare que la croissance hospitalière repose autant sur des

25 La croissance du système hospitalier américain au tournant du XIX ${ }^{\mathrm{e}}$ siècle est en effet impressionnante. On passe de 178 établissements (y compris les hôpitaux psychiatriques) et 50000 lits en 1873 à 4359 établissements et plus de 420000 lits en 1909. Rosenberg 1979.

26 Principalement Chandler 1977.

27 Goebel 1994.

28 Bridgman Perkins 1997, 420.

29 Shumsky 1978.

30 Starr 1982,148.

31 Sturdy 1992, 130.

32 Sturdy 1992, 128.

33 Cooter 1993.

34 Pickstone 1985.

35 Shumsky 1978, 542.

36 Howell 1995. 
aspects gestionnaires que techniques - innovations médicales proprement dites. Le grand mérite de l'ouvrage est en effet de montrer que l'arrivée d'un nouveau type de management permet de penser l'hôpital en terme de croissance: «L'attention croissante envers l'organisation a un impact direct sur la médecine clinique: les formulaires, les tableaux statistiques et une nouvelle structure institutionnelle ont permis et encouragé le développement de la chirurgie. ${ }^{37}$ Dans le même esprit, Paul Starr lie le développement des hôpitaux, ainsi que l'abandon de leur nature philanthropique, à l'affirmation de la profession médicale et déclare que «la chirurgie a rendu les soins hospitaliers rentables» ${ }^{38}$.

Il faut aussi souligner le travail novateur de David Rosner sur les établissements hospitaliers de New York dans les années 1885-1915 ${ }^{39}$ dont il étudie la modernisation et la réorganisation («hospital-standardization $»^{40}$ ). Il insiste notamment sur les changements démographiques, l'industrialisation et la réorganisation spatiale de la ville - et non sur les «progrès» de la médecine ${ }^{41}$ - pour expliquer la naissance d'hôpitaux de grande taille gérés selon les principes de l'économie privée ${ }^{42}$ qui deviennent un lieu de travail privilégié pour le corps médical. Rosner montre de manière tout à fait convaincante comment des institutions caritatives se transforment en entreprises rationalisées et dominées par le culte de l'«efficiency». Il met notamment en évidence le rôle de l'arrivée dans les conseils d'administration d'hommes issus des milieux économiques, aux côtés des philanthropes, et explique que la volonté de ces hommes nouveaux est «d'exercer une expertise financière et de sauver les hôpitaux, plutôt que de réaliser une réforme morale et sociale sur les patients» ${ }^{43}$. Morris Vogel s'est penché sur la professionnalisation des directeurs d'hôpitaux aux Etats-Unis dans les années $1895-1915^{44}$ et apporte ainsi un heureux complément à l'étude de Rosner.

37 Howell 1995, 66.

38 Starr 1982, 157.

39 Rosner 1982.

40 Rosner 1982, VIII.

41 «Les changements significatifs dans la forme de l'hôpital ont souvent eu lieu avant que de nouvelles techniques médicales soient introduites. Dans bien des cas, les hôpitaux ont été réorganisés pour des raisons internes avant que les avancées médicales et chirurgicales n'obligent à de quelconques réaménagements.» Rosner 1982, 7.

42 Le Dr Robert Dickinson, médecin établi à Brooklyn et impliqué dans la gestion hospitalière, déclare en 1917 que «le travail hospitalier n'est plus une entreprise caritative mais un business dont le travail peut être rationalisé de la même manière que d'autres entreprises commerciales». Cité par Rosner 1982, 61.

43 Rosner 1982, 53. Cette nouvelle conception de l'administration hospitalière ne va pas toujours de soi. En 1914, le président du Lutherian Hospital déclare ainsi que «notre travail à l'hôpital est un travail de secours. Ce n'est pas un business». Rosner 1982, 53.

44 Vogel 1989. 
Regroupés en une association professionnelle avec leurs collègues canadiens, qui compte plus de 450 membres en 1908, ces «superintendents» sont très engagés dans le mouvement tayloriste dont ils s'inspirent pour la conception de nouveaux bâtiments hospitaliers faisant la part belle à une médecine plus spécialisée et plus coûteuse, ainsi que pour l'uniformisation des plans comptables et des systèmes d'enregistrement des patients. Selon les sociologues Marc Berg et Stefan Timmermans, la standardisation de l'infrastructure hospitalière américaine du début du $\mathrm{XX}^{\mathrm{e}}$ siècle est la première étape d'un mouvement qui touche l'ensemble des pratiques médicales dans les années 1980 et 1990, et qui voit la généralisation d'une «evidence-based medicine» reposant sur des directives standardisées à une échelle généralement internationale ${ }^{45}$.

Pour Joan Lynaugh, auteur d'un ouvrage sur les hôpitaux de Kansas City dans les années 1870-1915, le changement d'échelle et la rationalisation des hôpitaux mettent fin au communautarisme ethnique et religieux qui était à la base de nombreux établissements ${ }^{46}$. La croissance hospitalière nécessite en effet le passage à la prise en charge en masse de malades - qui payent leur hospitalisation - ou à la recherche de filières-niches telles que la pédiatrie pour les établissements qui désirent conserver une taille restreinte. La fin de la philanthropie est ainsi «intimement liée à des pressions économiques sur les hôpitaux ${ }^{47}$. Cette perspective rejoint celle de Morris Vogel sur les hôpitaux de Boston, selon lequel le développement hospitalier repose en grande partie sur les classes moyennes, qui acceptent leur hospitalisation en échange de soins payants ${ }^{48}$. Pour le Canada, James M. Wishart a aussi montré que le développement hospitalier nécessite le recours à une clientèle aisée et rentable pour laquelle de nouveaux espaces sont aménagés ${ }^{49}$.

D'autres historiens anglo-saxons ont rédigé des contributions originales sur des aspects particuliers de la gestion hospitalière. L'organisation interne des établissements hospitaliers au début du $\mathrm{XX}^{\mathrm{e}}$ siècle a fait l'objet de plusieurs communications. George Rosen s'est intéressé à la réorganisation des établissements de soins ambulatoires américains (policliniques et dispensaires) dans les années 1900-1920 selon les principes du «scientific management $»^{50}$. Largement dépendants de la charité privée au XIX ${ }^{\mathrm{e}}$ siècle, les soins ambulatoires sont réorganisés dès les années 1900 dans le sens d'une meilleure rentabilité - et donc l'abandon de la gratuité généralisée - sous la pres-

45 Berg/Timmermans 2003.

46 Lynaugh 1989.

47 Lynaugh 1989, 175.

48 Vogel 1980.

49 Wishart 2001.

50 Rosen 1976. 
sion des médecins généralistes dénonçant la concurrence déloyale du système caritatif. Analysant la pratique de la médecine à Chicago dans les années 1900-1920, Thomas Goebel arrive aux mêmes conclusions ${ }^{51}$. Quant à Susan Reverby, elle s'intéresse aux essais de standardisation de la chirurgie du médecin de Boston Amory Codman au début du $\mathrm{XX}^{\mathrm{e}}$ siècle ${ }^{52}$. Le Dr Codman, assistant au Massachusetts General Hospital, lance au début des années 1900 un vaste programme de réforme de la médecine en milieu hospitalier fondé sur les méthodes de travail du secteur industriel. Voulant identifier les dysfonctionnements au sein de l'hôpital, il promeut la mise en place d'«efficiency committees» mais se heurte à la plupart de ses confrères qui refusent de voir leur propre travail évalué de la sorte. Certaines mesures de standardisation administrative seront toutefois reprises par des associations fédérales de médecins et de chirurgiens et diffusées par leurs canaux.

Citons encore les recherches de Stephen Kunitz sur la rationalisation du système sanitaire américain ${ }^{53}$ - principalement les assurances-maladie et de Steven Cherry sur les ressources hospitalières privées en GrandeBretagne $^{54}$. La problématique du financement hospitalier est aussi abordée depuis les années 1990 en dehors de la sphère anglo-saxonne, avec des travaux comme ceux de l'historien allemand Reinhard Spree sur le développement et le financement du système hospitalier allemand au cours du $\mathrm{XIX}^{\mathrm{e}}$ siècle $^{55}$ ou de l'Espagnol Pedro Pérez Castroviejo sur la gestion hospitalière dans le Pays basque dans les années 1800-1936 $6^{56}$. En France, il n'y a guère que l'ouvrage de Maurice Garden sur les finances des Hospices civils de Lyon aux XIX ${ }^{\mathrm{e}}$ et $\mathrm{XX}^{\mathrm{e}}$ siècles à aborder la dimension économique du développement hospitalier ${ }^{57}$. En Suisse aussi, quelques travaux récents abordent la question du financement et du management des hôpitaux ${ }^{58}$. Dans sa thèse sur les professions infirmières en Suisse romande, Joëlle Droux s'intéresse ainsi à «l'entreprise hospitalière $»^{59}$, à son organisation et à sa gestion, afin de mettre en lumière l'affirmation des diverses filières de professionnalisation du personnel hospitalier. Enfin, l'étude systémique du développement des

51 Goebel 1994.

52 Reverby 1981.

53 Kunitz 1981.

54 Cherry 1996, Cherry 1997 et Cherry 2000. Pour la Grande-Bretagne, un ouvrage de synthèse sur la question du financement hospitalier est annoncé: Gorsky/Sheard à paraître en février 2006 (avec notamment des articles de Steven Cherry et de Keir Waddington).

55 Parmi de nombreuses contributions, on retiendra sa synthèse Spree 1995 et l'édition de l'ouvrage collectif Labisch/Spree 2001.

56 Pérez Castroviejo 2002.

57 Garden 1980.

58 Voir Vouilloz Burnier/Barras 2004; Christ 1997; Christ 1999; Donzé 2000; Donzé 2003.

59 Droux 2000, 413. 
établissements hospitaliers vaudois a quant à elle permis de montrer que la modernisation médico-technique de leur infrastructure débouchait sur une situation de concurrence interhospitalière et de blocages financiers qui nécessitent en fin de compte l'intervention étatique ${ }^{60}$.

\section{Conclusion}

L'intégration des apports de l'histoire sociale des techniques et de l'histoire économique des hôpitaux dans l'explication du développement de la médecine hospitalière au cours des $\mathrm{XIX}^{\mathrm{e}}$ et $\mathrm{XX}^{\mathrm{e}}$ siècles ouvre d'intéressantes perspectives dans l'analyse historique du développement hospitalier. Le concept de système sociotechnique élaboré par Thomas Hughes s'avère en effet d'une excellente utilité pour aborder ce phénomène et montrer que la profonde réorganisation des hôpitaux à la fin du $\mathrm{XIX}^{\mathrm{e}}$ siècle et dans la première partie du $\mathrm{XX}^{\mathrm{e}}$ siècle s'apparente à une révolution industrielle, au sens où l'entend François Caron, c'est-à-dire au passage d'un système technique à un autre. Plusieurs types d'innovations, à la fois purement techniques (nouvelle manière de pratiquer la chirurgie), sociales (professionnalisation des médecins) et économiques (élargissement du marché des soins) favorisent alors le passage à un nouveau «système sociotechnique médical» fondé sur deux sous-systèmes principaux: le système hospitalier et le système technique médical. Il faut entendre par système hospitalier un ensemble d'acteurs institutionnels (hôpitaux publics et privés, cliniques, facultés de médecine, écoles d'infirmières, etc.) et sociaux (médecins, malades, hommes politiques, administrateurs, etc.), en interaction les uns avec les autres, et dépendant d'un environnement politique, économique et social commun. Le système hospitalier apparaît comme un élément structurant du marché médical, l'essentiel de l'offre de soins et d'infrastructures tendant à s'y concentrer dès la fin du XIX ${ }^{\mathrm{e}}$ siècle. Il peut alors s'organiser aussi bien d'après le principe de la concurrence et du libre marché que d'après celui d'un marché réglementé, voire cartellisé, selon l'intervention des divers acteurs - les constructeurs de système chers à Hughes - et leurs rapports de force au sein du système hospitalier. Quant au système technique médical, il faut l'entendre dans le sens de la manière dont se pratique la médecine, avec les divers modes de travail, les machines, les outils et les gestes techniques mis en œuvre dans l'acte de soigner, soit selon une acception proche de celle de Gille. Cependant, ce développement du système sociotechnique médical ne se réalise pas de manière

60 Donzé à paraître. 
harmonieuse et automatique: les blocages et les innovations sont omniprésents. L'évolution du système technique médical dépend étroitement de celle du système hospitalier, et vice versa. Les constructeurs de systèmes interviennent à tous les niveaux, selon des rapports de force variables dans le temps et l'espace. La transformation de la médecine hospitalière peut ainsi se concevoir sous des formes diverses, selon l'issue des conflits et des négociations qui permettent une réadaptation du système.

\section{Bibliographie}

Berg, Marc/Stefan Timmermans, The Gold Standard. The Challenge of Evidence-Based Medicine and Standardization in Health Care (Philadelphia 2003)

Bijker, Wiebe, Of Bicycles, Bakelites and Bulbs. Toward a Theory of Sociotechnical Change (London/Cambridge 1995)

Bijker, Wiebe/Thomas P. Hughes/Trevor Pinch (editors), The Social Construction of Technological Systems. New Directions in the Sociology and History of Technology (London/ Cambridge 1999 [11989])

Blume, Stuart S., Insight and Industry. On the Dynamics of Technological Change in Medicine (London/Cambridge 1992)

Bridgman Perkins, Barbara, "Shaping institution-based specialism: early twentieth-century economic organization of medicine", Social History of Medicine (1997) 419-435

Caron, François, Les deux révolutions industrielles du $20^{e}$ siècle (Paris 1997)

Chandler, Alfred, La main visible des managers. Une analyse historique (Paris 1988 [1 ${ }^{\mathrm{re}}$ éd. anglaise 1977])

Cherry, Steven, "Accountability, entitlement, and control issues and voluntary hospital funding c1860-1939", Social History of Medicine (1996) 215-233

- "Before the National Health Service: financing the voluntary hospitals, 1900-1939", Economic History Review (1997) 305-326

- "Hospital Saturday, workplace collections and issues in late nineteenth-century hospital funding", Medical History (2000) 461-488

Christ, Thierry, «De la bienfaisance privée à l'Etat social? Mise en place, financement et contrôle du réseau hospitalier et institutionnel (orphelinats, hospices) à Neuchâtel (1815-1914)», Revue historique neuchâteloise (1997) 23-51

- «Du zèle piétiste à l'activisme républicain. Démocratisation de la gestion et du contrôle de l'hôpital à Neuchâtel au 19 e siècle», Revue historique neuchâteloise (1999) 19-31

Cohen, Yves/Dominique Pestre, «Histoire des techniques»(dir.), Annales. Histoire, Sciences sociales (1998) 721-943

Cooter, Roger, Surgery and Society in Peace and War: orthopaedics and the organisation of modern medicine, 1880-1948 (London 1993)

- /Mark Harrison/Steve Sturdy (editors), Medicine and Modern Warfare (Amsterdam 1999)

Delamare, Jean/Thérèse Delamare-Riche, Le grand renfermement: histoire de l'hospice de Bicêtre, 1657-1974 (Paris 1990)

Dommann, Monika, Durchsicht. Einsicht. Vorsicht. Eine Geschichte der Röntgenstrahlen, 18961963 (Zürich 2003)

Donzé, Pierre-Yves, L'hôpital bourgeois de Porrentruy (Porrentruy 2000)

- Bâtir, gérer, soigner. Histoire des établissements hospitaliers de Suisse romande (Genève 2003)

- L'ombre de César. Les chirurgiens et la construction du système hospitalier vaudois (18401960) (à paraître en 2006)

Droux, Joëlle, L'attraction céleste. La construction de la profession d'infirmière en Suisse Romande (19e-20 siècles) (Université de Genève 2000) 
Faure, Olivier, Genèse de l'hôpital moderne: les hospices civils de Lyon de 1802 à 1845 (Lyon 1982)

- «Vingt ans d'histoire de la santé», Revue historique vaudoise (1995) 315-327

- «La médicalisation vue par les historiens», dans: Pierre Aïach/Daniel Delanoë, L'ère de la médicalisation. Ecce homo sanitas (Paris 1998) 53-68

Foucault, Michel, Naissance de la clinique (Paris 1963)

- «L'incorporation de l'hôpital dans la technologie moderne», dans: Dits et écrits, vol. 3 (1998) $508-521$

Garden, Maurice, Histoire économique d'une entreprise de santé: le budget des Hospices civils de Lyon, 1800-1976 (Lyon 1980)

Gille, Bertrand (dir.), Histoire des techniques (Paris 1978)

Goebel, Thomas, "American Medicine and the 'Organisational Synthesis': Chicago Physicians and the Business of Medicine, 1900-1920", Bulletin of the History of Medicine (1994) 639663

Gorsky, Martin/Sally Sheard, Financing Medicine. The British experience since 1750 (London, in press 2006)

Goubert, Jean-Pierre (éd.), La médicalisation de la société française, 1770-1830 (Waterloo 1982)

Howell, Joel, Technology in the Hospital. Transforming patient care in the early twentieth century (Baltimore/London 1995)

Hughes, Thomas P., Networks of Power: electrification in Western Society, 1880-1930 (Baltimore $\left.1993\left[{ }^{1} 1983\right]\right)$

- «L'histoire comme systèmes en évolution», Annales. Histoire, Sciences sociales (1998) 839_ 857

Imbert, Jean (dir.), Histoire des hôpitaux en France (Toulouse 1982)

Jones, Colin/Roy Porter (éd.), Reassessing Foucault: power, medicine and the body (London/ New York 1994)

Keel, Othmar, L'avènement de la médecine clinique moderne en Europe, 1750-1815 (Genève 2001)

Kunitz, Stephen, "Efficiency and reform in the financing and organization of American medicine in the progressive era", Bulletin of the History of Medicine (1981) 497-515

Labisch, Alfons/Reinhard Spree, «Neuere Entwicklungen und aktuelle Trends in der Sozialgeschichte der Medizin in Deutschland - Rückschau und Ausblick», Vierteljahrschrift für Sozial- und Wirtschaftsgeschichte (1997) 171-210 und 305-321

- (Hrsg.), Krankenhaus-Report 19. Jahrhundert. Krankenhausträger, Krankenhausfinanzierung, Krankenhauspatienten (Frankfurt 2001)

Latour, Bruno, Pasteur: guerre et paix des microbes (Paris 2001 [11984])

- /Steve Woolgar, Laboratory Life: the social construction of scientific facts (London 1979)

Léonard, Jacques, Les médecins de l'Ouest au XIX ${ }^{e}$ siècle (Paris 1978)

Loetz, Francisca, Von Kranken zum Patienten. «Medikalisierung» und medizinische Vergesellschaftung am Beispiel Badens 1750-1850 (Stuttgart 1993)

Löwy, Ilana (ed.), Medicine and Change: Historical and Sociological Studies of Medical Innovation (Paris/London 1993)

Lynaugh, Joan, The Community Hospitals of Kansas City, Missouri, 1870-1915 (New York/ London 1989)

Pérez Castroviejo, Pedro M., «La formación del sistema hospitalario vasco: administración y gestión económica, 1800-1936», Transportes, Servicios y Telecomunicaciones (2002) 73-97

Pickstone, John, Medicine and Industrial Society: a history of hospital development in Manchester and its region, 1752-1946 (Manchester 1985)

- Ways of Knowing. A new history of science, technology and medicine (Manchester 2000)

Reverby, Susan, "Stealing the Golden Eggs: Ernest Amory Codman and the science and management of medicine", Bulletin of the History of Medicine (1981) 156-171

Rosen, George, "The efficiency criterion in medical care, 1900-1920: an early approach to an evaluation of health service", Bulletin of the History of Medicine (1976) 28-44

Rosenberg, Charles, "Inward vision and outward glance: the shaping of the American hospital, 1880-1914", Bulletin of the History of Medicine (1979) 346-391

Rosner, David, A Once Charitable Enterprise. Hospitals and health care in Brooklyn and New York, 1885-1915 (Cambridge 1982) 
Schlich, Thomas, Surgery, Science and Industry. A Revolution in Fracture Care, 1950s-1990s (Basingstoke 2002)

Shumsky, Neil Larry, "The Municipal Clinic of San Francisco: a study in medical structure", Bulletin of the History of Medicine (1978) 542-559

Spree, Reinhard, «Krankenhausentwicklung und Sozialpolitik in Deutschland während des 19. Jahrhunderts», Historische Zeitschrift (1995) 75-105

Stanton, Jennifer, "Making sense of technologies in medicine", Social History of Medicine (1999) 437-448

- (ed.), Innovations in Health and Medicine. Diffusion and resistance in the twentieth century (London/New York 2002)

Starr, Paul, The Social Transformation of American Medicine. The rise of a sovereign profession and the making of a vast industry (New York 1982)

Sturdy, Steve, "The political economy of scientific medicine: science, education and the transformation of medical practice in Sheffield, 1890-1922", Medical History 36 (1992) 125-159

Ueyama, Takahiro, "Capital, profession and medical technology: the electro-therapeutic institutes and the Royal College of Physicians, 1888-1922", Medical History (1997) 150-181

Vogel, Morris J., The Invention of the Modern Hospital: Boston 1870-1930 (Chicago/London 1980)

- "Managing medicine: creating a profession of hospital administration in the United States, 1895-1915", in:Lindsay Granshaw/Roy Porter (eds), Hospitals in History (London/New York 1989) 243-260

Vouilloz Burnier, Marie-France/Vincent Barras, De l'hospice au réseau santé. Santé publique et systèmes hospitaliers valaisans, $X I X^{e}-X X^{e}$ siècles (Sierre 2004)

Wishart, James M., "Class difference and the reformation of Ontario Public Hospitals, 1900-1935: "Make Every Effort to Satisfy the Tastes of the Well-to-Do"', Labour/Le Travail 48 (2001) 27-62 